\title{
AUTOMOTIVE THEFT DETECTION USING FACE RECOGNITION
}

\author{
Shejina $\mathbf{V}^{1}$, Asil $\mathbf{A}^{2}$ \\ ${ }^{1}$ Assistant Professor, Department of Information Technology, College of Engineering, Vadakara, Kerala, India \\ ${ }^{2} Q A$ Engineer, Nucore Software Solutions Pvt Ltd, Ernakulam, Kerala, India
}

\begin{abstract}
Nowadays vehicles are becoming an important part of human life. Vehicles have a tremendous role on lightening the life styles of people. At the same time the rate of vehicle theft is increasing day by day. Here comes the importance of detection of vehicle theft. The existing security system is based on anti theft alarm. Whenever a person touch the body of the vehicle, the alarm is automatically turned on and sounds. The limitation of the existing system is that an intruder can break the security alarm when he is able to access the power source. The aim is to develop a security system which can be incorporated inside a vehicle for the purpose of detecting theft. The proposed system is a face recognition based security system with face recognition module loaded in Raspberry Pi. When the driver sits in the driver seat and inserts key in to the key hole, a sensor is activated and a hidden camera captures the image of the driver which is then sent to the Raspberry Pi through an RS-232 cable. The captured image is pre-processed using histogram equalization. The pre-processed image is given for face detection. Face detection is performed using Viola-Jones algorithm. The extracted face is recognized using the Local Binary Patterns Histograms algorithm. If the vehicle is stolen, the software sends an mms to the owner comprising the image of the driver and the current location of the vehicle. The system is well implemented in OpenCV-Python and trained to identify two possible drivers. The software can distinguish between authorized and unauthorized drivers and send email to the owner if the vehicle is driven by an unauthorized person.
\end{abstract}

Keywords: Raspberry Pi B+, Histogram Equalization, Viola-Jones algorithm, Local Binary Patterns Histogram

\section{INTRODUCTION}

Building up of a security mechanism that is incorporated inside an automotive is a novel idea. The proposed system is having great relevance in detecting theft of a vehicle when the owner parks his vehicle in a parking lot or anywhere. The idea of authenticating a vehicle using face recognition system incorporated inside Raspberry Pi is a novel one. The main advantage of face recognition among other biometric methods is that it does not require the active co-operation of a person and can be used in cases where people are unknown about the presence of face recognition system.

Here we used Raspberry $\mathrm{Pi} \mathrm{B}+$ as the microcomputer in which the face recognition module is loaded. It is a small, low cost computer developed by Raspberry Pi foundation ,UK. It is a single board computer which is capable of doing any work a desktop can do and can be placed anywhere inside the vehicle.

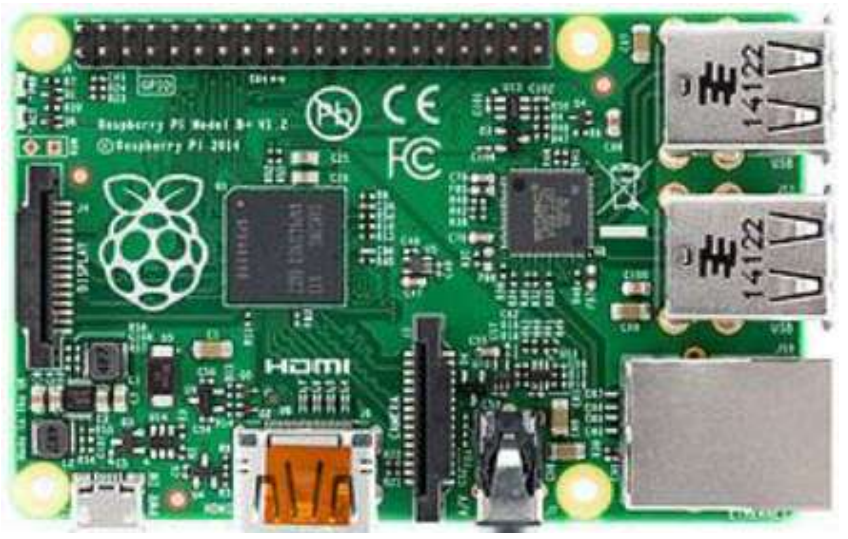

Fig -1: Raspberry Pi B+

\section{BLOCK DIAGRAM}

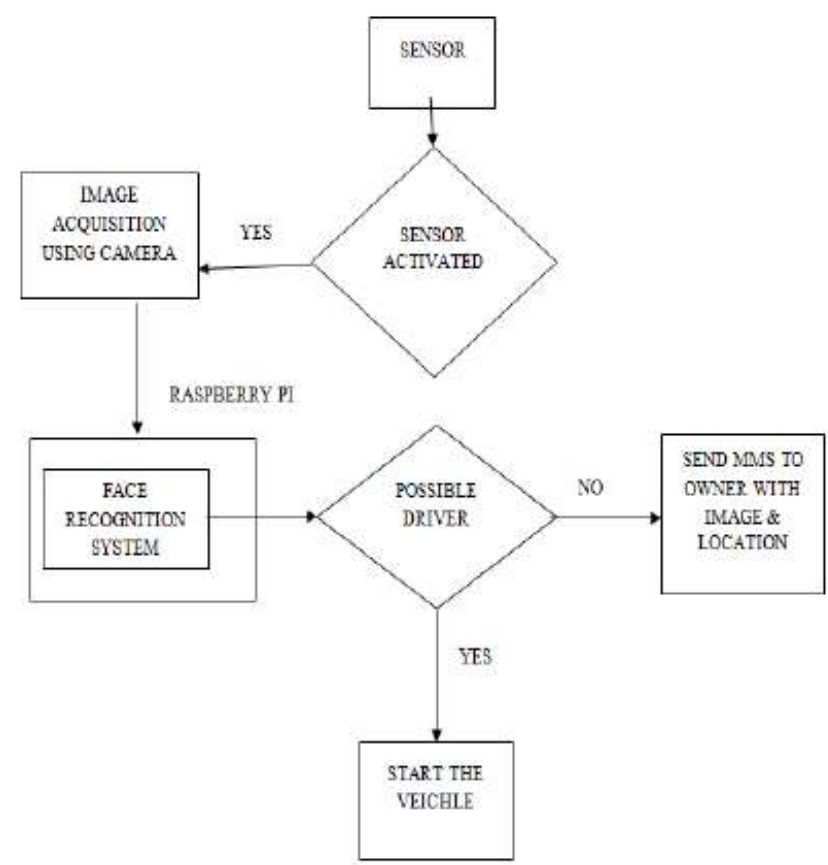

Fig -2: Block Diagram

The image of the driver ,captured using a camera is passed to the face recognition system loaded into the Raspberry Pi. The face recognition system detects the face portion in the image and compares it with the images of possible drivers in the training set. If a mismatch is found the owner is informed of the vehicle theft by sending an mms containing the acquired image of the driver as well as the current location of the vehicle. 


\section{MODULE DESCRIPTION}

The software has three modules.

- Image Acquisition Module

- Face recognition Module

- Owner Module

\subsection{Image Acquisition Module}

When a person enters into the vehicle and put the key into the key hole a sensor will be activated which in turn activates a hidden camera in the vehicle. The camera will capture the image of the driver and pass it to the microcomputer through an RS-232 cable for further processing.

\subsection{Face Recognition Module}

The face recognition module is loaded into the Raspberry Pi $\mathrm{B}+$ for processing of the captured image. The captured image is preprocessed using histogram equalization to better adjust the intensity values on the histogram. It is then passed to the face recognition module. The module comprises processes such as face detection and face recognition.

\subsubsection{Face Detection}

Face detection identifies human faces in digital images. The process of extracting the face blob from its surroundings and localization of its exact position is referred to as face detection. It finds or marks the region belonging to a face in an image. In this system Viola-Jones algorithm [1] is used for detecting and extracting face blob.

In Viola-Jones algorithm, the input image is partitioned into a number of sub windows. From these sub windows we are extracting certain features that corresponds to human face. The algorithm consists of four stages.

- Haar features selection

- Creating integral image

- AdaBoost Training algorithm

- Cascaded classifiers

The input image captured by the camera consists of portions other than face blob. Here we have to extract the face blob from its surroundings by discarding areas other than the face in the image. There are certain properties that can be used for identifying a human face from other portions in an image. These properties are matched using Haar Features. The properties that are common to human faces are:

- The eyes region tends to be more darker than the upper cheeks

- The nose bridge is more brighter than other regions in the face

This knowledge can be used to construct matching facial features by identifying the location and size of eyes, mouth $\&$ nose bridge region and calculating the gradient of pixel intensities. Basically three kinds of features such as tworectangle feature [1], three-rectangle feature [1] and fourrectangle feature [1] are computed.
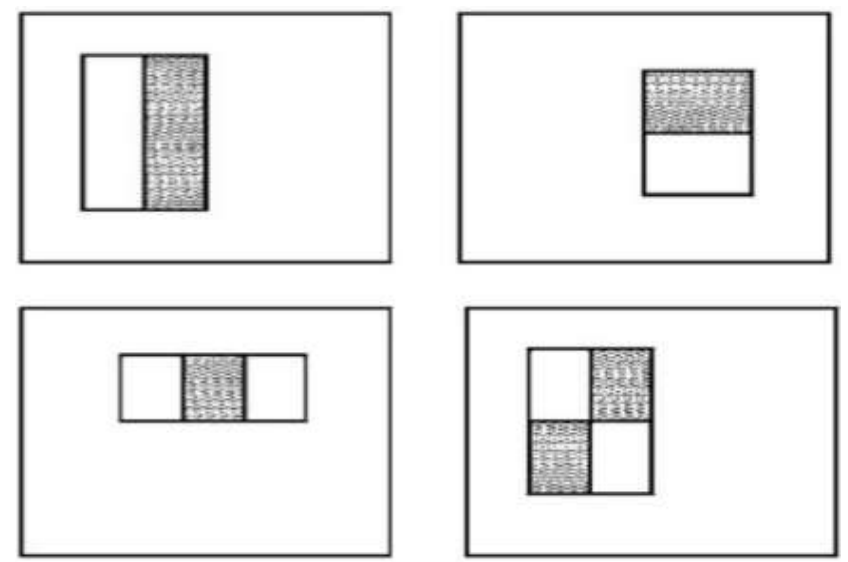

Fig -3: Two rectangle, Three rectangle and Four rectangle feature

The resolution of the base detector is $24 \times 24$ and the set of features extracted is quite large. So we change the image into an integral image. This is done by making each pixel equal to the sum of all pixels above it and to the left of it.

Now we have collected the feature vector. The next step is to select the best features to train the classifier. This is done by the AdaBoost algorithm by considering the facial properties. Now we will change the size of the detector and continue the above procedures with a new window size. There may be large number of evaluated sub windows which consists of regions not belonging to a face. So the algorithm should reject non-faces and the cascaded classifier [1] is used to do this task.

\subsubsection{Face Recognition}

Face recognition is the process of identifying or verifying a person in the given digital image .In recognition phase, the extracted face blob is matched with the images of possible drivers in the training set. If a match is found, the face in the given digital image is recognized. Otherwise an mms is sent to the owner of vehicle including the details of the vehicle theft.

Here face recognition is achieved by extracting Local Binary Patterns[2][4] from the face blob. In this method, the face blob is divided into cells of $16 \times 16$ each. Each pixel in the cell is compared with its eight neighbors. If the intensity of the center pixel is lesser than its neighbor, then denote it with 0 and 1 if not. So, for each pixel, we will get a binary number, just like 11101001 . So with 8 surrounding pixels we have $2^{\wedge} 8$ possible combinations, referred to as LBP codes. The operator takes the $3 \times 3$-neighbourhood of each pixel and thresholds it with the central value and there by forms a binary number. We can ake the histogram of these labels which can be used as a texture descriptor.

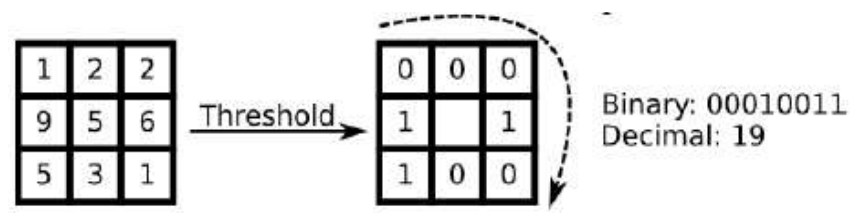

Fig -4: A basic LPB operator 
An LBP operator can be defined as:

$$
\operatorname{LBP}\left(x_{c}, y_{c}\right)=\sum_{p=0}^{p-1} 2^{p} s\left(i_{p}-i_{c}\right)
$$

, with $\left(\mathrm{x}_{\mathrm{c}}, \mathrm{y}_{\mathrm{c}}\right)$ as central pixel with intensity $\mathrm{i}_{\mathrm{c}}$; and $\mathrm{i}_{\mathrm{n}}$ being the intensity of the neighbor pixel. is the sign function defined as:

$$
s(x)= \begin{cases}1 & \text { if } x \geq 0 \\ 0 & \text { else }\end{cases}
$$

Then create circular neighborhoods by aligning an arbitrary number of neighbors on a circle with a variable radius, as shown in Fig-5.

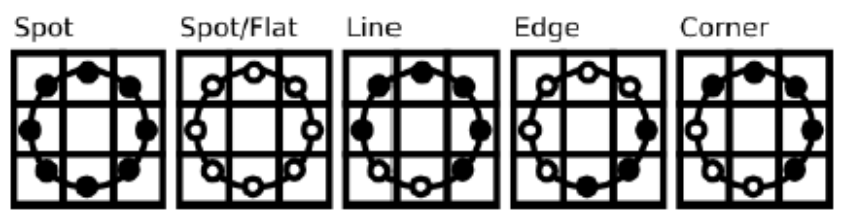

Fig -5: Circular neighborhood

The created LBP image is divided into $k$ local regions to find out the histograms. All the local histograms are then concatenated to generate a feature vector. These histograms are called Local Binary Patterns Histograms. In this histogram, we effectively have a description of the face.

\subsection{Owner Module}

The owner module comprises the process of sending mms to the owner when an unauthorized person tries to drive the vehicle. The output of face recognition module is used for this purpose. When a person who is not authorized tries to drive the vehicle, an mms containing the image of the person and the current location of the vehicle is sent to the owner. The owner can then inform the police about the theft with a solid proof.

\section{RESULTS}

The training set consists of four different frontal images of two possible drives authorized to drive the vehicle as shown in Fig-6 .This is fed to the face recognition system and it is trained to identify the possible drivers.
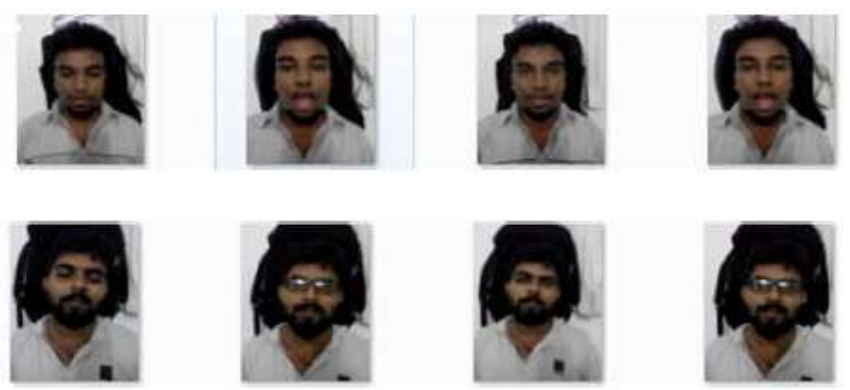

Fig -6: The training set
The system very well identifies the possible drivers if they enter in to the vehicle. If an unknown person such as shown in Fig-7 enters in to the vehicle, face recognition system recognizes him as a thief and informs the owner about the vehicle theft.

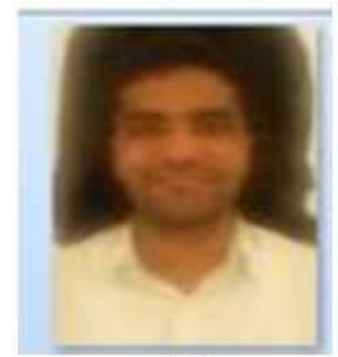

(a)

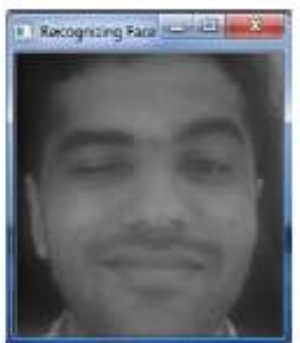

(b)
Fig -7: (a) Image of an unauthorized person (b) Recognized Face

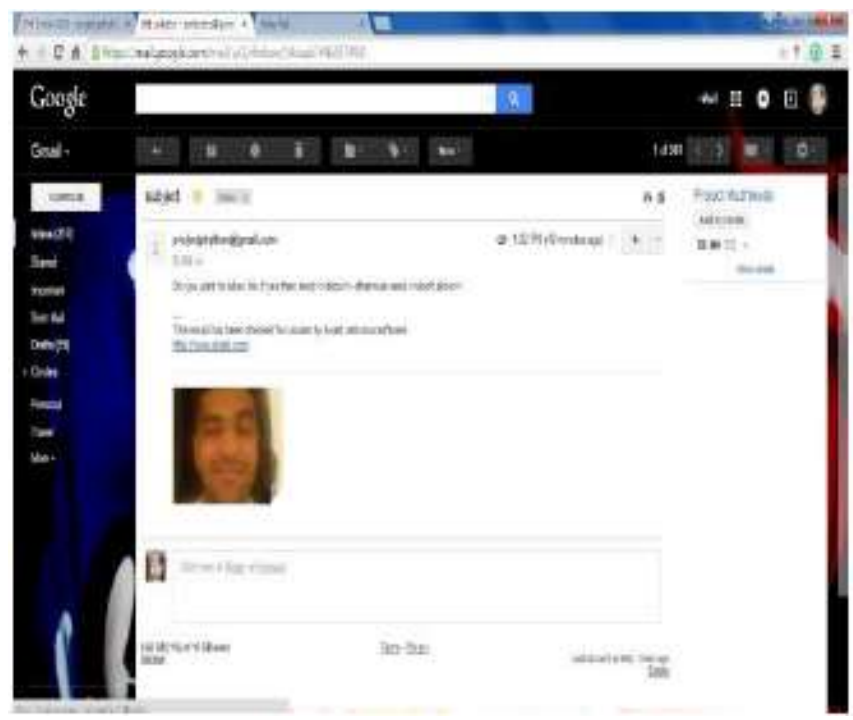

Fig - 8: Screen shot of the email sent to owner

\section{CONCLUSION}

The proposed system is a novel idea that enables the manufacturer of a vehicle to embed the security system inside the vehicle at the time of manufacturing. Here it is illustrated how face recognition can be used in the detection of vehicle theft. For face detection we used Viola-Jones algorithm and Local Binary Patterns Histogram method for face recognition. The two methods are efficiently implemented in Open CV along with Python. Viola Jones algorithm is a fast method of detecting face regions from images. The Local Binary Patterns Histogram method successfully classified images as authorized and unauthorized and email is sent to the owner when the person is unauthorized.

\section{REFERENCES}

[1]. Paul Viola, Michael J. Jones," Robust Real-Time Face Detection", International Journal of Computer Vision 57(2), 2004. 
[2]. Sarabjit Singh1, Amritpal Kaur2, Taqdir, "A Face Recognition Technique Using Local Binary Pattern". International Journal of Advanced Research in Computer and Communication Engineering,Vol. 4, Issue 3, March 2015

[3]. C. Nandakumar,G. Muralidaran and N. Tharani, "Real Time Vehicle Security System Through Face Recognition “,International Review of Applied Engineering Research, ISSN 2248-9967 Volume 4, 2014

[4] Timo Ahonen ,Abdeneour Hadid,Matti Pietikinen,"Face Recognition with Local Binary Patterns",Macine Vision Group,University of Finland

[5]. V.Balajee Seshasayee, E Manikandan, "Automobile Security System Based on Face Recognition Structure Using GSM Network", Advance in Electronic and Electric Engineering. ISSN 2231-1297,Volume 3,Number 6(2013)

\section{BIOGRAPHIES}

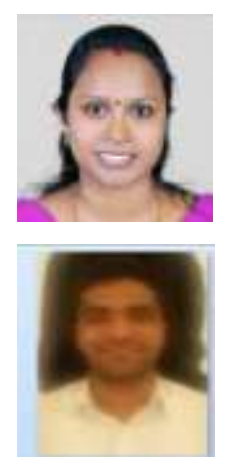

Mrs Shejina V has been working as an Assistant Professor in Dept. of Information Technology, College of Engg, Vadakara ,Calicut, Kerala.

Mr Asil A has been working as a QA Engineer in Nucore Software Solutions Pvt Limited, Ernakulam, Kerala 